\title{
Diffractive Optics
}

\author{
Yunlong Sheng \\ University Laval \\ Center for Optics, Photonics and Lasers \\ Department of Physics, Physical \\ Engineering and Optics \\ Sainte-Foy, Quebec, Canada G1K 7P4 \\ E-mail: sheng@phy.ulaval.ca
}

\section{Pierre Ambs}

Université de Haute-Alsace

\section{ESSAIM}

\section{Laboratoire MIPS}

12, rue des Frères Lumière

68093 Mulhouse Cedex, France

E-mail: p.ambs@uha.fr

Diffractive and micro optics have had fast growth in the last 20 years since microlithography technology began to be used for fabrication of optical elements. Nowadays, micro and diffractive optical element (DOE) fabrication has reached a certain maturity. A wide range of applications has been developed. New applications and new integrated diffractive optical systems continue to emerge. The 22 papers from 16 countries published in this special section on diffractive optics reflect the recent trends and progress in DOE applications, fabrications, optical system design and implementations, and subwavelength structures. Both of us have witnessed and contributed to the progress of diffractive optics technology and have been co-chairing SPIE conferences on diffractive optics at Photonics Asia and the SPIE Annual Meeting, respectively. It is a real pleasure for us to jointly edit the first special section on diffractive optics in Optical Engineering.

The recent advances in nanoscale lithography and holography techniques provide a huge potential to create new subwavelength structures that efficiently manipulate light through diffraction and propagation. We believe that the new nanofabrication technologies will once again push diffractive optics and its natural extensions, including subwavelength structures, photonic crystals, near-field optics and surface plasmon polariton optics, etc., to a new fast advance path. The impact will be even more important than what has happened in the last 20 years by the application of microlithography to fabrication of DOEs.

In this special section on diffractive optics, new DOE applications include surface plasmon resonance sensors, ultrashort-pulse processing, solar energy systems, interferometric testing, high-power laser beam shaping, dynamic lenses, fiber Bragg grating fabrication, 3-D chipscale optical interconnections, and Talbot array illumination.
Pedersen et al. design and imprint near-field surface relief diffractive gratings for beam deflection and focusing in an injection-moulded polymer chip for the new integrated surface plasmon resonance sensor, which is lowcost, compact, and robust with high performance. Lohokare et al. develop a novel conductive polymer-based flip-chip integration process for packaging arrayed VCSELs and microlenses, and demonstrate a 3-D chip-scale optical interconnection system. Grunwald et al. present the spatiotemporal processing of an ultrashort-pulse laser beam by exploiting specific advantages of thin-film micro-optical arrays. Gombert et al. address the design and the whole experimental process for the fabrication of surface-relief microstructures on large areas that are useful in solar energy systems for antireflective surfaces, displays, light trapping in polymer solar cells, and facade protection from sun radiation. Pruss et al. apply computer-generated holograms, which are able to generate several aspheric wave fronts, in interferometer metrology. The system is used for absolute testing of aspheric surfaces and diffractive transmission spheres. For highpower laser beam shaping applications, Liu et al. develop a modified iterative Fourier transform design algorithm. They design the beam shaping DOE and the DOE that combines beam conditioning and fan-out functions for coupling the beam into multimode optical fibers. Ripoll et al. present a review and compare some useful iterative Fourier transform algorithms for the design of continuous and multiphase-level DOEs for beam shaping.

In the next three papers, Fresnel near-field diffraction is studied for new applications. Onural discusses mathematical properties of the sampled quadratic phase function (chirp), mainly that the Fourier transform of a sampled chirp function is also a sampled chirp function. The results are used in computer simulation of digital 
Fresnel diffraction. Wang and Zhou present theoretical analysis on multilevel phase gratings for Talbot array illumination at partial Talbot distances for potential applications to optical scanners and holographic storage. For an important application of phase gratings for fabrication of fiber Bragg gratings, Sheng et al. model the e-beam phase mask with stitching errors and analyze its effect on sidelobes in the fiber grating spectrum using the Talbot formula.

For micro and diffraction optical element fabrication, Rudmann and Rossi review wafer scale replication and UV embossing technologies for mass production of micro-optical components. Replications of double-sided high-quality components for data communication applications and single-side low-cost components for consumer electronic applications are demonstrated. Lee et al. report on the design, fabrication, and characterization of $3 \lambda$ period blazed grating composed of subwavelength ridges of progressively varying width for thermal imaging in the 8 - to $12-\mu \mathrm{m}$ wavelength band. He et al. report the design and fabrication, with reflow and reaction ion etching, of a microlens array, which is monolithically integrated on the back of the substrate of an infrared focal plan array. Du et al. present simplified exposure dose equations for controlling the profile of microlenses in a thick photoresist layer. Refractive lens arrays with aspherical profile and high filling factor are fabricated with this technique. Using the research facility in the Centre Spatial de Liège for producing large-size (up to $380 \mathrm{~mm}$ ) dichromated gelatin volume phase holographic gratings, Blanche et al. have recorded diffraction gratings of high angle performance, close to $100 \%$ diffraction efficiency, and less than 5\% angular deviation of efficiency at low temperatures down to $93^{\circ} \mathrm{K}$ for astronomical infrared (IR) applications. Sokolova et al. describe a new two-step recording of concave diffraction gratings, which uses the aspheric wave front from the first spherical concave gratings. The final grating is recoded in the spatially incoherent light. Shcherbakov et al. investigate theoretically the possibility of implementing all-optical digital logic by using the phenomenon of three-order light diffraction with direct transitions between all the optical modes in uniaxial single crystals.

For subwavelength structures, Lenaerts et al. designed passive guided mode resonance filters under a 45-deg incidence for wavelengths close to $633 \mathrm{~nm}$ and active substrate mode electro-optic modulators. The 45-deg incidence reduces effectively the reflection coefficient in TM polarization.

For full complex amplitude modulation DOEs, Neto et al. develop a device that consists of cells whose amplitude is modulated by changing the light transmission area and the phase is modulated in the remaining area. Tudela et al. implement full complex amplitude Fresnel holograms using two liquid crystal devices extracted from a video projector. They show the wave front reconstruction at several distances using different approaches of encoding.

In a technical note, Lu studies the nonlinear relationship of the photoresist height and the optical density of the HEBS photomask of high-resolution gray steps $(<10$ $\mu \mathrm{m})$ in order to calibrate the micro-optic fabrication process with precise surface relief profiles. The dependence of the photoresist height profile on the width of the gray step is shown, which agrees well with the simulation result from the model. A Fresnel DOE based on the technique is manufactured. In another technical note, Loukina et al. present in detail the analysis, design, trade-off consideration, and experimental results for volume phase gratings in the application as a dispersive element in telecom spectral equalizers.

We would like to thank all those who made this special section on diffractive optics possible, especially the authors for all the submitted papers and the reviewers for their time and effort. Our special thanks go to Lisa Polinder, Kristi Kelso, Anne Munger, Karolyn Labes, and other SPIE staff for working with us to make this special section a reality. We want to thank Prof. Donald O'Shea, the Editor of Optical Engineering, for his support and help.

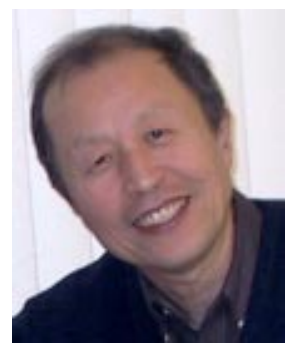

Yunlong Sheng received the BS degree from the University of Sciences and Technology of China in 1964. He received the MSc, Doctor, and Doctor d'Etat degrees in physics from the Université de Franche-Comte, Besançon, France, in 1980, 1982, and 1986, respectively. Since 1985, he has been with the Centre for Optics, Photonics and Lasers, University Laval, and is now a full professor. Dr. Sheng has been an author or co-author of more than 180 technical papers, books, and book chapters and holds two patents on fiber Bragg grating fabrication. His research interests involve diffractive optics, fiber Bragg gratings, nanooptics, and image science. Dr. Sheng is a member of OSA and a fellow of SPIE-The International Society for Optical Engineering.

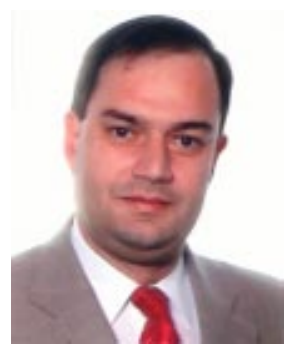

Pierre Ambs is a professor of electrical engineering at the Université de Haute Alsace, Mulhouse, France. His research interests include optical and computergenerated diffractive optical elements, optical pattern recognition, and optical storage and their applications to optical processing and computing. Dr. Ambs received his MS (1979), PhD (1982), and Doctorate d'Etat (1987) from the Université de Haute Alsace. He is a member of SPIE, OSA, the European Optical Society, and the Sociéte Française d'Optique. 